\title{
Apoio social e o cuidado integral à saúde do idoso
}

\section{I ${ }^{1}$ Marcelo Barbosa Otoni Gonçalves Guedes, ${ }^{2}$ Kenio Costa Lima, \\ ${ }^{3}$ Célia Pereira Caldas, ${ }^{4}$ Renato Peixoto Veras I}

Resumo: O crescente envelhecimento

populacional e as transformaçôes socioculturais do nosso tempo conduzem a novas necessidades, não somente de cuidado, mas também para atender a uma nova perspectiva do conceito de saúde, no qual cuidar da doença se tornou ação limitada. Este estudo trata-se de um ensaio baseado na literatura científica e se propóe a pensar sobre uma pequena parte desta grande questão: o apoio social como parte da atenção integral à saúde do idoso. Para isso, realizou-se uma revisão narrativa da literatura nas bases de dados Pub Med/MedLine, Crochane e Scopus, bem como nas listas de referências dos artigos encontrados. Parte-se da compreensão do papel do apoio social e das redes microssociais na saúde do idoso. A seguir, discutimos formas de avaliar o apoio e a rede social e refletimos sobre o papel do apoio social no Sistema Único de Saúde. Por fim, aborda-se o manejo do apoio social. Como consideraçôes finais, são destacados os principais desafios para que o Apoio Social seja realmente considerado um determinante da saúde das pessoas.

> Palavras-chave: apoio social; saúde da pessoa idosa; integralidade em saúde.

\author{
1 Universidade Federal \\ do Rio Grande do Norte. \\ Pós-Graduação em Saúde \\ Coletiva. Natal-RN, Brasil \\ (marcelloguedes21@hotmail. \\ com). \\ ${ }^{2}$ Universidade Federal do \\ Rio Grande do Norte. Pós- \\ Graduação em Saúde Coletiva. \\ Natal-RN, Brasil (limke@uol. \\ com.br). \\ ${ }^{3}$ Universidade do Estado do \\ Rio de Janeiro, Universidade \\ Aberta da Terceira Idade. Rio de \\ Janeiro-RJ, Brasil (celpcaldas@ \\ gmail.com). \\ ${ }^{4}$ Universidade do Estado do \\ Rio de Janeiro, Universidade \\ Aberta da Terceira Idade. Rio de \\ Janeiro-RJ, Brasil (unativeras@ \\ gmail.com).
}

Recebido em: 15/02/2017 Aprovado em: 08/07/2017 


\section{Introdução}

O envelhecimento populacional tem gerado novas demandas para os sistemas de saúde públicos e privados em todo o mundo. $\mathrm{O}$ caminho para o cuidado integral parece ainda náo estar claro para os profissionais da saúde, gestores e para os usuários de nossos sistemas de saúde. Para elucidar esta questão, faz-se necessária a discussão sobre abordagens multidimensionais para o cuidado que considerem uma nova perspectiva do conceito de saúde, sob uma ótica mais ampla (PAIM; ALMEIDA-FILHO, 2014).

O envelhecer está presente nas agendas de vários fóruns em todo o mundo. A grande preocupação dos que discutem o tema reside na violação da garantia dos direitos sociais próprios dessa parcela da populaçáo. $\mathrm{Na}$ grande maioria das sociedades, o "ficar velho" é sinônimo de exclusão de uma vida social, construída e legitimada ao longo dos anos. No entanto, a lógica populacional tem pressionado o surgimento de uma preocupação voltada aos que vivem essa fase da vida.

Para a área da saúde, o processo de envelhecimento se caracteriza por sua complexidade, para o qual é necessária uma abordagem interdisciplinar, buscando associar conteúdos das ciências médicas, sociais, da Psicologia e conhecimentos político-geográficos, entre outros, exigindo de seus profissionais uma qualificação específica para o tratamento deste segmento da população (MEDINAWALPOLE et al., 2001; STAMM, 2001). Dessa forma, uma avaliação restrita às condiçóes patológicas do ponto de vista biológico restringe uma ação ampla do contexto de saúde na população, sobretudo nos idosos, como clientela vulnerável no acesso aos serviços de saúde de qualidade e resolutivos. Como consequência, percebemos hoje açóes reducionistas que negligenciam avaliaçóes e intervençóes integradas aos aspectos psicossomáticos, sociais, médicos e culturais.

Segundo o IBGE (BRASIL, 2012), os idosos consomem, per capita, 63 reais com gastos com internação, valores estes que se elevam a 179 reais quando se trata de idosos acima de 80 anos. O maior consumo de recursos do sistema de saúde se deve às constantes internaçóes, ao maior tempo de ocupação do leito e às muitas patologias que os afetam. Corroborando esta preocupação, de acordo com o aumento cada vez maior da populaçáo envelhecida, a Organizaçáo Mundial da Saúde (OMS) defendeu a saúde dos idosos como uma das prioridades entre as inúmeras questóes no âmbito sanitário. Açóes de intervenções mais amplas, em um nível de atenção primária à saúde, poderiam se tornar mais resolutivas, 
trazendo melhor assistência e menores custos ao sistema de saúde (PAIM et al., 2011; CIOSAK et al., 2011). Pesquisas apontam, por exemplo, para uma associação direta entre indicadores relacionados a diversas condiçôes patológicas ou adversas às condiçóes de vida e aspectos sociais negativos, como é o caso do suporte social inadequado (KIM, 2012; MELCHIORRE et al., 2013; NERI; VIEIRA, 2013).

No Brasil, o documento das diretrizes do Pacto pela Saúde, publicado por meio da Portaria n 399/GM, em fevereiro de 2006 (BRASIL, 2006a), que contempla o Pacto pela Vida, inclui, em um dos seus pontos, a defesa da saúde dos idosos como uma das seis prioridades pactuadas entre as três esferas de governo. São apresentadas açóes que objetivam pôr em prática algumas diretrizes da Política Nacional de Saúde da Pessoa Idosa, tomando como referência os princípios e diretrizes do Sistema Único de Saúde (BRASIL, 2006b).

O Apoio Social se enquadra como parte importante da atenção integral à saúde do idoso, sendo definido como a integração do suporte emocional, financeiro, instrumental e relacionamento social que pessoas ou instituiçóes possam oferecer, neste caso, para os idosos (MELCHIORRE et al., 2013; NERI; VIEIRA, 2013; MARQUES et al., 2013; GONÇALVES, 2011). Muitos são os autores que afirmam ser o suporte social um dos aspectos mais relevantes quando se pensa em melhorias de condições de vida e de saúde das pessoas, incluindo as idosas (FREITAS et al., 2016; JOHNSON, 2014; LETCHER; PERLOW, 2009) e que sua importância aumenta com o decorrer do envelhecimento (MAIA et al., 2016). A falta de rede social é preditora de mortalidade por diversas causas (HOBBS et al., 2016; ZUNZUNEGUI et al., 2004). Relatos de melhoria de parâmetros médicos, como saúde mental (LADITKA et al., 2009), depressão (SEHLO; KAMFAR, 2015; MARQUES et al., 2013; BAPTISTA; BAPTISTA; TORRES, 2006; CHI; CHOU, 2001; MUI, 1996), incapacidades (JAMES et al., 2011), parâmetros sociais como prevenção à institucionalização (RODRIGUES; SILVA, 2013; KRISTJANSSON; BREITHAUPT; McDOWELL, 2001; BARKER, 2002) e parâmetros de saúde como bem-estar (PAZIN et al., 2016; KUTEK; TURNBULL; FAIRWEATHER-SCHMIDT, 2011; KENDIG; BROOKE, 1997) e qualidade de vida (FERNANDEZ-MAYORALAS et al., 2012; BARKER; MORROW; MITTENESS, 1998) estão entre os mais estudados e reforçam o quanto uma rede social adequada em sua extensão e qualidade de relaçóes alicerçam o suporte em nível emocional, instrumental, informacional e cognitivo (GONÇALVES et al., 2011; FONTES, 2004). 
Uma rede social que fornece um apoio inapropriado pode estar relacionada com diversos aspectos tão amplos, que extrapolam condiçóes patológicas e poderia estar associada diretamente a indicadores de mortalidade, levando não somente a uma população idosa sob maior risco e vulnerabilidade, mas também apresentando risco iminente de questóes extremamente preocupantes na populaçáo jovem e adulta, como é o exemplo dos casos de tendência a suicídios em populaçóes que geralmente estão sob efeito de alguma condição de risco à saúde e que possuem níveis de apoio social inadequados (FÉLIX et al., 2016; OMS, 2014; KLEIMAN; LIU 2014). Diante do que foi exposto, nosso objetivo é discutir aspectos importantes do Apoio Social para o cuidado integral à saúde do idoso, como seu processo de avaliação, manejo, importância das redes microssociais e a relação com o Sistema Único de Saúde (SUS).

\section{Metodologia}

Para atender aos propósitos do estudo, procedemos com um ensaio baseado em pressupostos construídos a partir de levantamento bibliográfico. A revisão da literatura foi do tipo narrativa, com pesquisa na internet, entre os meses de outubro e dezembro de 2016; os critérios de busca e inclusão dos artigos estáo descritos na tabela 1 . Os cruzamentos entre os descritores eram sempre realizados entre "social support" e um segundo descritor, utilizando o operador boleano "and". Em seguida, as informaçôes foram compiladas em formato de temáticas para facilitação do entendimento.

Tabela 1. Critérios de busca e inclusão de artigos científicos referentes ao Apoio Social, Natal-RN, Brasil, 2017

\begin{tabular}{ll}
\hline Período da publicação & 1996 a 2016 \\
\hline Base de dados & $\begin{array}{l}\text { Pub Med/MedLine; Crochane; Scopus; Busca ativa } \\
\text { nas referências dos artigos encontrados }\end{array}$ \\
\hline Descritores & $\begin{array}{l}\text { Social Support; Aged; Handling; Evaluation; Social } \\
\text { Networking; Public Health }\end{array}$ \\
\hline Operador boleano & "and" \\
\hline Restritores & Seres humanos com 60 anos ou mais \\
\hline Idiomas & Inglês, Português, Espanhol \\
\hline Total de artigos incluídos & $\mathbf{6 0}$ \\
\hline
\end{tabular}


Geib (2012) destaca as redes sociais e comunitárias como determinantes sociais definitivos para a saúde dos idosos, definindo-as como capital social formado por relaçóes fortalecidas por confiança, cooperação e reciprocidade. Portanto, redes sociais são fortalecidas quando existe reciprocidade nas relaçôes interpessoais. Quando ela não existe, a interação social se desequilibra, enfraquecendo os laços da coesão social e, consequentemente, das relaçôes sociais, formando assim um ciclo prejudicial para a vida das pessoas em fase de vida mais avançada.

Para muitas pessoas idosas, as redes constituem o único recurso disponível para aliviar as cargas da vida cotidiana e aquelas que provêm da enfermidade (GEIB, 2012), trazendo suporte emocional e uma percepção sobre si mesmo mais positiva (GONÇALVES, 2011), numa concepção que vai muito além do simples fato de ir à farmácia ou à padaria como forma de ajudar, mas sim de envolver o idoso efetiva e afetivamente num ambiente positivo e acolhedor.

Destacados alguns pontos sobre o papel da rede e do apoio social para os idosos, acreditamos importante a discussão sobre as seguintes questôes: Como são constituídas as redes microssociais? Quem seriam os atores sociais envolvidos e que poderiam dar suporte à construção desta rede? Para sermos breves, enfatizaremos os seguintes entendimentos: a) existem pessoas mais próximas, com contatos sociais frequentes, por exemplo, uma vez na semana ou algumas vezes no mês, e normalmente estão presentes em situaçóes de necessidade, esses atores sociais formam nossa rede microssocial (MAIA et al., 2016; AMARAL, 2013); b) esta rede pode também ser composta por profissionais qualificados, como os da saúde, por exemplo, ou serviços estatais, os quais poderão fornecer apoio social, neste caso, do tipo formal, ou por amigos, parentes, vizinhos, que também poderão fornecer um suporte social, neste caso, do tipo informal (MAIA et al., 2016; MARTINS, 2005). É interessante deixar claro que a rede microssocial pode ser composta hora por apoio formal, hora por apoio informal.

Alvarenga et al. (2011), estudando uma população de 503 idosos da cidade de Dourados no Mato Grosso do Sul, constataram que a rede mais próxima de contatos sociais para o grupo era constituída em importância decrescente principalmente por filhos, vizinhos, netos e amigos. Nesse mesmo estudo, outros atores também foram citados, como agentes comunitários de saúde e irmãos. Maia et al. (2016), em estudo desenvolvido com 306 idosos em Portugal, 
1190 identificaram os familiares e rede de amigos como os maiores preditores para o envelhecimento ativo.

Um importante aspecto destacado por Gardner (2011), no que diz respeito ao suporte informal, é a constituição das redes naturais de vizinhança (Natural Neighborhood Networks- $N N N$ ), que compreendem três tipos de relaçôes. Relaçóes de proximidade (vizinhos), relaçôes de serviço (motoristas de táxi, funcionários de lojas, entre outros) e relaçóes com pessoas que não residem no bairro. Tais redes melhorariam sobremaneira o suporte oferecido pela família e amigos e não substituiria o suporte formal, mas o complementaria.

Barker (2002) destaca que a relação existente entre os cuidadores informais sem parentesco com o idoso caracteriza um cuidado casual, limitado, cuja relação é comprometida. Tais relaçôes sofrem variação de acordo com o grau de afetividade entre os cuidadores e idosos. Ainda assim, o autor ressalta que essas relaçóes são benéficas, podendo reduzir o grau de ansiedade dos idosos fragilizados e evitar a institucionalizaçáo.

Sabe-se, no entanto, que existe certa complexidade na avaliação das redes microssociais, já que elas vão além de simplesmente identificar quem poderiam ser as pessoas envolvidas, fazendo-se necessário o entendimento de aspectos importantes, como a extensão da rede e a qualidade do apoio recebido pelos atores nela envolvidos. A relaçáo social de cuidado desenvolvida pelo idoso com a família, amigos ou até mesmo outros idosos se torna algo benéfico a partir do momento em que existe o bem-estar do idoso, o qual se configura por meio do bom relacionamento com a família, amigos e vizinhos (KENDIG \& BROOKE, 1997).

O papel da rede de suporte ofertada por amigos, família e vizinhos para idosos canadenses frágeis foi avaliado por Fast et al. (2004). Segundo os autores, as redes de cuidados podem variar muito em função do tamanho, da composição da relação, gênero, idade e proximidade. Portanto, as características da rede podem vir a expor os idosos a riscos de receber suporte inadequado. Pequeno tamanho da rede, altas proporçóes de cuidadores não parentes, homens e membros distantes geograficamente e falta de cônjuge ou companheiro(a) em casa são alguns possíveis fatores associados ao suporte inadequado.

Efeitos negativos do suporte social na saúde e bem-estar também são descritos. Ramos (2002) cita que pode haver efeito negativo na autoestima do idoso que recebe suporte, quando este o reconhece como dependência e falta de autonomia, 
e, mais ainda, quando existe falta de capacidade de retribuir o auxílio recebido.

Muitos indivíduos podem se deprimir diante de uma troca náo balanceada. Relaçóes descompassadas entre os atores envolvidos no suporte ofertado e recebido também podem gerar efeito negativo.

Questôes relacionadas a gênero sempre permeiam a discussão do suporte informal. Em vários estudos (BOURQUE et al., 2005; BARRETT; LYNCH, 1999; BARKER; MORROW; MITTENESS, 1998), as mulheres apresentam mais cuidados informais que os homens. A figura da filha adulta é o elementochave no cuidado informal às mulheres. Quanto aos homens, especialmente aqueles casados com mulheres que não são as mães dos seus filhos, tendem a ter redes de apoio pequenas e vulneráveis (BARKER; MORROW; MITTENESS, 1998). Larsson e Thorslund (2002), no entanto, afirmam que os homens são mais propensos a receber cuidado informal através de suas esposas. Segundo eles, as mulheres, por sua vez, recebem mais cuidados do tipo formal. Para eles, é muito mais comum as mulheres viverem sozinhas. Os homens moram com a esposa ou outro familiar, o que implica uma maior prevalência de recebimento de cuidados pelos homens. No estudo de Larsson e Thorslund (2002), 54\% dos homens recebiam cuidado informal de um morador do mesmo domicílio. Para as mulheres, esse valor era de $13 \%$. Segundo eles, não houve diferença no recebimento de cuidado informal entre pessoas de nível educacional diferente.

O suporte para idosos náo casados e sem filhos foi estudado por Wu e Pollard (1998). Uma vez que existem funçôes sociais específicas de filhos, nestes idosos estas costumam ser realizadas pelos irmãos, que podem ter senso de compromisso similar ao existente na relação entre pais e filhos. O subgrupo dos viúvos e divorciados parece estar sob maior risco de falta de suporte. Os solteiros, ao contrário, tendem a cultivar mais amizades de maneira mais ativa ao longo da vida, e estas poderão funcionar como rede de suporte no envelhecimento. Segundo os autores, o apoio oferecido pelos vizinhos está mais relacionado a situaçôes emergenciais, tendo em vista a proximidade geográfica. Por outro lado, o apoio prolongado é provido por parentes.

\section{Avaliaçáo do apoio social e da rede social}

Para avaliação do apoio social, uma série de questóes é proposta em vários estudos. Os aspectos mais relevantes nesse sentido englobam uma avaliação da 
composição e tamanho da rede social, a durabilidade, a proximidade geográfica, a frequência do contato social, a intimidade e a reciprocidade, a qualidade do suporte social recebido, o suporte instrumental e emocional e a ajuda aos outros (GOMIDE; SCHÜTZ, 2015; THANAKWANG et al., 2011; WALTERGINZBURG, 2002; CHI; CHOU, 2001).

Algumas ferramentas específicas podem ser usadas para avaliar o suporte informal e, consequentemente, analisar a rede em que o idoso está inserido, mostrando-se instrumentos importantes para pesquisas e avaliação da situação de saúde para intervençóes que venham a contemplar os diversos aspectos de saúde do idoso, incluindo os sociais. Nosso objetivo aqui não é aprofundar nossos esforços no entendimento desses instrumentos, mas citaremos brevemente alguns para que nossos leitores possam em momento oportuno buscar mais conhecimento sobre eles. É, também, do entendimento dos presentes autores, que necessitamos de instrumentos com critérios de construção e validação mais cuidadosos (AERA, APA, NCME, 2014), e que acolham a população brasileira nos seus aspectos culturais e sociais específicos. Ainda assim, a utilização dos instrumentos disponíveis no momento, poderá direcionar o profissional para uma avaliação menos arbitrária e, consequentemente, um manejo do apoio social mais eficaz. No quadro abaixo (quadro 1), segue um resumo com alguns instrumentos:

Quadro 1. Instrumentos para avaliação do Apoio Social, ano de publicação e abordagem da pesquisa. Natal-RN, 2017.

\begin{tabular}{|l|l|l|}
\hline Instrumento/Autor & Ano & Abordagem \\
\hline $\begin{array}{l}\text { Escala de apoio } \\
\text { social para hábitos } \\
\text { alimentares } \\
\text { saudáveis } \\
\text { (PESSINI et al.) }\end{array}$ & 2016 & $\begin{array}{l}\text { Avaliou a equivalência transcultural e as propriedades } \\
\text { psicométricas em pessoas de 24 a } 86 \text { anos. O } \\
\text { instrumento apresentou equivalência transcultural e } \\
\text { propriedades psicométricas adequadas para aplicaçáo em } \\
\text { adultos e idosos. }\end{array}$ \\
\hline $\begin{array}{l}\text { Mapa Mínimo } \\
\text { de Relaçóes do } \\
\text { Idoso: análise de } \\
\text { reprodutibilidade } \\
\text { (MMRI) } \\
\text { (DOMINGUES) }\end{array}$ & 2000 & $\begin{array}{l}\text { Apresenta a reprodutibilidade do MMRI (versão de } \\
\text { adaptaçáo transcultural de 2000 do mesmo autor), que é } \\
\text { constituído por quatro quadrantes que representam família, } \\
\text { amigos, comunidade e relaçóes com os serviços sociais ou } \\
\text { de saúde. Sobre esses quadrantes inscrevem-se três áreas, } \\
\text { ou seja, um círculo interno de relaçóes mais próximas, um } \\
\text { intermediário e outro de contatos ocasionais. }\end{array}$ \\
\hline
\end{tabular}

continua... 


\begin{tabular}{|c|c|c|}
\hline $\begin{array}{l}\text { Escala de percepção } \\
\text { de suporte social } \\
\text { (EPSS) (Siqueira } \\
\text { M.M.M.) }\end{array}$ & 2008 & $\begin{array}{l}\text { Aplicada a pessoas com média de idade de } 23,93 \text { anos. } \\
\text { A partir de análise fatorial, obteve-se relevantes índices } \\
\text { psicométricos. }\end{array}$ \\
\hline $\begin{array}{l}\text { Escala de apoio } \\
\text { social para a prática } \\
\text { de atividades físicas } \\
\text { (Easaf) (REIS et al.) }\end{array}$ & 2011 & $\begin{array}{l}\text { Avalia a validade e a fidedignidade da versão brasileira da } \\
\text { escala de apoio social para a prática de atividades físicas } \\
\text { em adultos brasileiros. }\end{array}$ \\
\hline $\begin{array}{l}\text { Escala de Suporte } \\
\text { Social para Pessoas } \\
\text { Vivendo com HIV/ } \\
\text { Aids (SEIDL; } \\
\text { TRÓCCOLI) }\end{array}$ & 2006 & $\begin{array}{l}\text { A análise fatorial exploratória indicou a existência de dois } \\
\text { fatores de primeira ordem: suporte social emocional e } \\
\text { suporte social instrumental. }\end{array}$ \\
\hline $\begin{array}{l}\text { Escala de Apoio } \\
\text { Social do Estudo } \\
\text { Pró-Saúde, } \\
\text { adaptada de Social } \\
\text { Support Survey of the } \\
\text { Medical Outcomes } \\
\text { Study (MOS) } \\
\text { (GRIEP et al.) }\end{array}$ & 2005 & $\begin{array}{l}\text { Composta por itens que abrangem cinco dimensões } \\
\text { funcionais de apoio social: "material”, "afetivo", } \\
\text { "emocional", "informação" e "interação social positiva". }\end{array}$ \\
\hline $\begin{array}{l}\text { Escala de Apoio } \\
\text { Social de Bille- } \\
\text { Brahe (EAS/ } \\
\text { BB) (GASPARI; } \\
\text { BOTEGA) }\end{array}$ & 2002 & $\begin{array}{l}\text { Questôes que avaliam nível de demanda do indivíduo } \\
\text { por apoio e de obtenção do auxílio de sua família e } \\
\text { amigos. }\end{array}$ \\
\hline $\begin{array}{l}\text { Escala de Suporte } \\
\text { Social e Estresse } \\
\text { na Infância e } \\
\text { Adolescência } \\
\text { (CUPERTINO) }\end{array}$ & 2001 & $\begin{array}{l}\text { Os autores estudaram o efeito do suporte social na } \\
\text { infância e adolescência sobre o estado depressivo de } \\
\text { idosos. }\end{array}$ \\
\hline $\begin{array}{l}\text { Questionário de } \\
\text { Suporte Social de } \\
\text { Norbeck (NSSQ) } \\
\text { (ANDRIOLA } \\
\text { et al.) }\end{array}$ & 1990 & $\begin{array}{l}\text { Avalia as múltiplas fontes de apoio social percebidas através } \\
\text { de três componentes funcionais (afeto, afirmação e ajuda) } \\
\text { e de três propriedades da rede social do sujeito (número de } \\
\text { pessoas componentes da rede, duraçáo dos relacionamentos } \\
\text { e frequência de contato com os membros da rede). }\end{array}$ \\
\hline
\end{tabular}




\section{Apoio social e o Sistema Único de Saúde: um ponto para reflexáo}

Um ponto que julgamos merecer reflexão refere-se a como as redes sociais estão inseridas no sistema de saúde público vigente no Brasil, o SUS. Considerando a integralidade um dos princípios finalísticos do nosso sistema de saúde, onde uma de suas dimensões é a observância do conceito ampliado em saúde, que deve dar atenção não só aos aspectos de doença, mas também aos sociais e psicológicos (PAIM; ALMEIDA-FILHO, 2014), o que vemos muitas vezes é que nossos serviços estáo preparados para atenderem a uma demanda somente quando se refere a doenças, sobretudo as de condiçóes agudas, de forma fragmentada e sem levar em conta o protagonismo e autonomia do usuário. Isso gera limitaçóes no atendimento, redução de eficácia e incremento nos gastos, sobretudo com aqueles que apresentam condiçóes crônicas, sejam patológicas ou de fatores de risco (MENDES, 2010), como é o caso da presença de uma rede microssocial desarranjada. Consideremos esta como uma condição de risco crônica, por se tratar de uma condição que afeta a vida de muitos idosos de forma continuada e prolongada. Nesse contexto adverso, as redes sociais bem arranjadas favoreceriam o suporte social e, consequentemente, levariam a uma redução de demanda nas redes de atenção à saúde.

No documento, Diretrizes para o cuidado das pessoas com doenças crônicas nas redes de atenção à saúde e nas linhas de cuidado prioritárias, elaborado pelo Ministério da Saúde (BRASIL, 2013), são preconizadas açóes voltadas para os determinantes sociais, lançando mão de ferramentas que sejam capazes de avaliar o processo saúde de forma completa, considerando a complexidade e singularidade de cada um e o meio onde ele está inserido. Em alguns momentos, aquele indivíduo mesmo medicado continua com seus níveis de pressão arterial elevados, e alguns profissionais atuando na atenção primária em saúde, ainda com uma visão hospitalocêntrica e meramente prescritiva, não entendem que podem existir diversos fatores, incluindo os sociais, que poderiam estar prejudicando a evolução do processo de tratamento, como é o caso das redes sociais. São sugeridas ainda nesse documento instrumentos e açóes atuais e integrativas para o sistema e para o sujeito, como o projeto terapêutico singular, a clínica ampliada, a atenção multiprofissional, o apoio matricial, o cuidado coletivo, o cuidado apoiado por leigos, o acompanhamento a distância e o autocuidado apoiado (BRASIL, 2013), que na prática não ocorrem nem com as condiçóes patológicas mais corriqueiras nem com as condiçóes sociais. 
Estamos cientes que introduzir essas novas ideias de atenção à saúde requer um esforço extra não somente das autoridades políticas, dos gestores dos serviços e profissionais envolvidos, mas também um esforço dos próprios usuários por uma responsabilidade compartilhada no cuidado de sua saúde ou das pessoas próximas, proporcionando o empoderamento e autonomia popular para as questóes de saúde e bem-estar do dia a dia deles mesmos. Este esforço deve ainda se desenvolver com ações intersetoriais envolvendo os diversos setores da sociedade, sejam eles da saúde, da educação, do meio ambiente, da segurança pública, do transporte, entre outros. Como estas açóes não ocorrem com a devida frequência, percebemos então um discurso do século XXI e as ações propriamente ditas com o cuidado à saúde, do século XIX, afastando-se, portanto, da integralidade objetivada pelo SUS. Assim sendo, além desta nova visão, discutiremos a seguir algumas sugestôes específicas para o manejo do suporte social e construçáo das redes microssociais, como alternativas de cuidado e forma de apoio às redes de atenção à saúde, que poderão ser inseridas nos diversos níveis dos serviços de saúde.

\section{O manejo do apoio social: estratégias e ferramentas baseadas em evidência}

$\mathrm{O}$ manejo do apoio social pode também apresentar efeito reverso positivo, ou seja, promover benefício aos que o executam. Estratégias como o autocuidado apoiado, a gestáo de cuidado por pares, os grupos operativos presenciais e não presenciais de idosos e o uso de ferramentas interativas via internet, como as redes sociais e aplicativos de conversas com smartphones, podem se mostrar importantes instrumentos multiplicadores do AS na comunidade e fortalecedores das redes microssociais. Citaremos nos próximos parágrafos algumas experiências exitosas em pesquisas e relatos com ações promotoras de apoio social formal e informal.

O manejo do apoio social por parte dos profissionais de saúde é incipiente. Em geral, os profissionais lançam mão do suporte formal. Muitas são as iniciativas de capacitaçáo dos próprios idosos como multiplicadores em programas de prevenção de doenças crônicas, através das comunidades ou instituiçóes religiosas. Destaca-se aqui o papel das "Promotoras de Saúde", mulheres idosas, identificadas por diversas instituiçóes religiosas e que foram capacitadas para atuarem em suas comunidades, levando à populaçáo conhecimentos, atitudes e formas de prevenção de vários tipos de cânceres. Entretanto, no que diz respeito a aspectos sociais, como é o caso do AS, essas açôes são ainda limitadas. 
Davis et al. (1998) avaliaram tais benefícios sobre idosos livres de doenças terminais ou transtornos cognitivos que receberam ou náo suporte ao cuidado por um ano, prestado por mentores voluntários idosos (mais jovens e mais saudáveis que os participantes do programa). Estes foram treinados durante 12 sessōes de duas horas, duas vezes na semana por seis semanas em relação a exercício, nutrição, segurança doméstica, fumo, uso do álcool e medicações. Ao final do estudo, observou-se que os voluntários não só prestaram serviços de apoio a outros, como também se beneficiaram (melhorias na saúde e função), indicando uma importância para a reciprocidade no Apoio Social para a saúde.

Barr e Russell (2007) documentaram as características das redes sociais de idosos residentes de um resort costeiro, como parte de um estudo qualitativo do seu capital social. Os autores destacaram que para a maioria dos respondentes, a aposentadoria foi o tempo de transição em que eles se mudaram para o resort costeiro, o que significou uma quebra dos padróes de conexão social. As famílias estavam geograficamente distantes e novas amizades necessitaram ser estabelecidas na área. A filiação a clubes ou associaçôes pode ser um dos caminhos nos quais as conexôes são feitas.

Outra forma proposta de manejo do suporte informal por parte dos idosos se dá através da participação desse idoso em programas sociais, incluindo-os nas escolas, por exemplo, a fim de que os mesmos possam contribuir na educação infantil (FRIED et al., 1997). Seguindo essa mesma linha de raciocínio, Frick et al. (2004) avaliam o custo-efetividade da experiência "CORPS" desenvolvida em Baltimore - EUA, onde os idosos têm a oportunidade de contribuir com seu trabalho "voluntário" nas escolas públicas, desenvolvendo atividades de moderado envolvimento físico, emocional e cognitivo. Os resultados demonstram uma melhoria na saúde e qualidade de vida dos tais "voluntários", a partir do conceito de suporte fornecido como ferramenta de valorização do próprio apoio social, e, consequentemente, redução dos gastos médicos.

A presença de idosos em grupos, ou seja, a sua própria mobilização na rede informal, tem sido vista em várias experiências exitosas. Um dos bons exemplos é a participação dos idosos cubanos no que Fernández-Alfonso, Pérez e Rodriguez (2007) denominam de "Círculo de Abuelos" amigos do esporte. Trata-se de uma organização comunitária que incentiva idosos a praticar atividades físicas e que tem trazido melhorias aos que delas participam. 
A formação de "pontes" entre idosos através dos recursos da informática também é uma das experiências que demonstram como o próprio idoso pode mobilizar a rede de suporte informal. Segundo Cornwell (2009), uma boa saúde física e principalmente cognitiva está relacionada com o arranjo de pontes sociais que proporcionem um envolvimento em um ambiente social refletido como uma ação positiva para a saúde integral dos idosos. Esta ação independe da idade dos idosos, mas sofre influência da experiência de vida, como o luto (positivamente) e problemas com a aposentadoria e saúde (negativamente). Hobbs et al. (2016) perceberam um risco de mortalidade reduzido entre idosos que realizam uma maior integração social em redes sociais como o Facebook.

Uma forma muito interessante de potencializar o AS por parte dos idosos é a descrita por Letcher e Perlow (2009). Trata-se do que eles denominam de "Community Exchange", ou comunidade de troca. A comunidade de troca usa a lógica do banco de horas ("time bank"), onde os indivíduos recebem créditos pelo tempo dedicado ao serviço de apoio. O suporte informal é constituído por variados tipos de açóes de acordo com as necessidades de quem vai recebê-las e com o conhecimento de quem vai ofertá-las, como ensinar a tocar um instrumento, conversar por telefone com quem não pode sair de casa, entre outras. Para quem oferta o seu tempo, a participaçáo nesse tipo de atividade aumenta o bem-estar e a autoestima, e para quem a recebe há uma contribuição com a promoção da saúde. Em nível coletivo, este modelo de suporte social baseado em parcerias colabora para o estabelecimento do respeito ao outro e redução de iniquidades. A reciprocidade é a palavra-chave desse trabalho voluntário.

Seguindo a linha de criação de grupos comunitários para o Apoio Social, Hale (2011) descreve em seu trabalho a prática da jardinagem comunitária, o significado e a história dos jardins, os aspectos sociais da jardinagem em comunidade e os relacionamentos estabelecidos com essa atividade. Segundo os autores, os indivíduos que participam das hortas comunitárias trabalham em todo o processo de plantação (preparo do solo à colheita), favorecendo o contato social, a aquisição de novos conhecimentos e as condiçôes de saúde, uma vez que eles se alimentam dos vegetais que cultivam.

A mobilização da rede de suporte informal deve transcender aspectos meramente de "uso" do suporte familiar, de vizinhos ou amigos e permitir o 
envolvimento dos idosos em atividades sociais que os conectem com a rede social de forma que ocorra uma interaçáo recíproca. Nessa perspectiva, um aspecto importante ressaltado por Ashida e Heaney (2008) é que suporte social e conexão social devem ser considerados construtos diferentes. Eles afirmam que indivíduos podem receber suporte social e apresentar sentimento de solidão. Portanto, eles sugerem que se deve associar o suporte informal à conexão social, garantindo assim o bem-estar do indivíduo. Tal ideia é reforçada por James et al. (2011), que defendem que a falta de interação social está associada à incapacidade. $\mathrm{O}$ mecanismo subjacente a essa relação é pouco conhecido.

\section{Considerações finais}

$\mathrm{Na}$ atualidade, as exigências pessoais sobre as necessidades do cuidado com a saúde se transformaram. A disponibilidade e o acesso a novas tecnologias e açôes para a saúde modificaram a percepção das pessoas e sociedade sobre como os serviços e os profissionais da saúde e de outras áreas se organizam e atuam. Entretanto, novas atitudes compatíveis com estas transformações são indispensáveis. As novas demandas e os anseios da população, sobretudo a idosa, só serão atendidos com uma mudança no olhar para um cuidar humanizado, efetivo, para e com estas pessoas.

Os desafios são enormes, entre eles, fazer perceber o Apoio Social como determinante importante da saúde das pessoas não somente nos serviços de saúde (seja este serviço inserido em qualquer nível de complexidade ou tecnológico), mas também na comunidade, como instrumento transformador do processo saúdedoença do idoso, devendo ser realizado pelos diversos atores sociais envolvidos, incluindo-se o próprio idoso e demais membros da sociedade (familiares, amigos, vizinhos, grupos religiosos, profissionais de saúde e do serviço social, estudantes, entre outros).

O apoio social é apenas uma das multifacetas que devem ser consideradas nesta nova perspectiva da atenção à saúde e não mais somente da atenção à doença. Mesmo sendo apenas parte de uma atenção integral, as redes microssociais podem ter um efeito multiplicador, nos aspectos sociais, psicossomáticos e, por que não dizer, biológicos das pessoas, proporcionando maior interação, reduzindo efeitos danosos à saúde e favorecendo o bem-estar dos idosos e daqueles que os rodeiam. ${ }^{1}$ 


\section{Referências}

ALVARENGA, M. R. M. et al. Rede de suporte social do idoso atendido por equipes de Saúde da Família. Cienc. Saúde Coletiva, v. 16, n. 5, p. 2.603-2.611, 2011.

AMARAL, F. L. J. S. et al. Apoio social e síndrome da fragilidade em idosos residentes na comunidade. Ciência \& Saúde Coletiva. Rio de Janeiro, v. 18, n. 6, p. 1.835-1.846, 2013.

AMERICAN EDUCATIONAL RESEARCH ASSOCIATION (AERA). American Psychological Association (APA). National Council on Measurement in Education (NCME). The standards for educational and psychological testing. New York: American Educational Research Association, 2014.

ANDRIOLA, W. B.; TROCCOLI, B.T.; DIAS, M.R. Caracterização do apoio social em estudantes universitários brasileiros. Rev Psicol, 7/8:61-78, 1990.

ASHIDA, S.; HEANEY, C.A. Differential associations of social support and social connectedness with structural features of social networks and the health status of older adults. J. Aging Health, v. 20, n. 7, p. 872-893, 2008.

BARKER, J. C.; MORROW, J.; MITTENESS, L. S. Gender, informal social support networks, and elderly urban African Americans. J. Aging Stud., v. 12, n. 2, p. 199-222, 1998.

BAPTISTA, M. N; BAPTISTA, A. S.; TORRES, E. C. Associação entre suporte social, depressão e ansiedade em gestantes. Psic Rev Vetor Ed, 7:39-48, 2006.

BARKER, J. C. Neighbors, friends, and other nonkin caregivers of community-living dependent elders. J. Gerontol., v. 57, n. 3, p. S158-167, 2002.

BARR, F.; RUSSELL, C. Social capital among older residents of a coastal resort: A survey of social resources and vulnerabilities. Australas. J. Ageing, v. 26, n. 2, p. 94-96, 2007.

BARRETT, A. E.; LYNCH, S. M. Caregiving networks of elderly persons: variation by marital status. Gerontologist, v. 39, n. 6, p. 695-704, 1999.

BOURQUE, P. et al. Contextual effects on life satisfaction of older men and women. Can. J. Aging, v. 24, n. 1, p. 31-44, 2005.

BRASIL. Ministério da Saúde. Agência Nacional de Saúde Suplementar (ANS). Plano de cuidado para idosos na saúde suplementar. Rio de Janeiro: ANS, 2012. 62p.

. Ministério da Saúde. Secretaria de Atenção à Saúde. Departamento de Atenção Básica. Brasília-DF. Diretrizes para o cuidado das pessoas com doenças crônicas nas redes de atenção à saúde e nas linhas de cuidado prioritárias. 2013.

. Portaria 2.528, de 19 de outubro de 2006. Aprova a Política Nacional de Saúde da Pessoa Idosa. Brasília: Diário Oficial da República Federativa do Brasil. Brasília, 2006b.

Portaria 399/GM, de 22 de fevereiro de 2006. Divulga o Pacto pela Saúde 2006 Consolidação do SUS e aprova as Diretrizes Operacionais do referido Pacto. Brasília: Diário Oficial da República Federativa do Brasil. Brasília, 2006a. 
CHI, I.; CHOU, K.L. Social support and depression among elderly Chinese people in Hong Kong. Int. J. Aging Hum. Dev., v. 52, n. 3, p. 231-252, 2001.

CIOSAK, S. I. et al. Senescência e senilidade: novo paradigma na Atenção Básica de Saúde. Rev Esc Enferm. USP, v. 45, esp. 2, p. 1.763-8, 2011.

CORNWELL, B. Network bridging potential in later life life-course experiences and social network position. J. Aging Health., v. 21, n. 1, p. 129-154, 2009.

CUPERTINO, A. P. Tradução da escala do inglês para o português: estudo dos processos de envelhecimento saudável [resumo]. Juiz de Fora (MG): Editora da Universidade Federal de Juiz de Fora, 2001.

DAVIS, C. et al. Benefits to volunteers in a community-based health promotion and chronic illness self-management program for the elderly. J. Gerontol. Nurs., v. 24, n. 10, p. 16-23, 1998.

DOMINGUES, M. A. Mapa mínimo de relaçôes: adaptação de um instrumento gráfico para a configuração da rede de suporte social do idoso. 2000. 120 f. Dissertação (Mestrado em Saúde Pública) - Faculdade de Saúde Pública, Universidade de São Paulo, São Paulo, 2000.

DOMingUES, M. A. Mapa Mínimo de Relaçôes do Idoso: análise de reprodutibilidade. Revista Kairós Gerontologia. São Paulo, v. 14, n. 6, p. 153-166, dez. 2011.

FAST, J. et al. Characteristics of family/friend care networks of frail seniors. Can. J. Aging., v. 23, n. 1, p. 5-19, 2004.

FÉLIX, T. A. Fatores de risco para tentativa de suicídio: produção de conhecimento no Brasil. Revista Contexto \& Saúde, v. 16, n. 31, p. 172-85, 2016.

FERNÁNDEZ-ALFONSO, J. M. F.; PÉREZ, L. D.; RODRIGUEZ, B. B. Life quality associated with health in the Elder's Club Sports Friends. Municipality of Matanzas 2006. Rev. Medica Eléctron, v. 29, n. 6, 2007.

FERNANDEZ-MAYOR ALAS, G. et al. Design, measures and sample characteristics of the CadeViMa-Spain survey on quality of life in community-dwelling older adults. Int. Psychogeriatr., v. 24, n. 3, p. 425-438, 2012.

FONTES, B. A formação do capital social em uma comunidade de baixa renda. Redes: Rev. Hispana Anal. Redes Soc., v. 7, n. 2, p. 1-33, 2004.

FREITAS, R.P.A. et al. Impacto do apoio social sobre os sintomas de mulheres brasileiras com fibromialgia. Rev Bras Reumatol. 2016.

FRICK, K. D. et al. Modeled cost-effectiveness of the experience corps Baltimore based on a pilot randomized trial. J. Urban Health, v. 81, n. 1, p. 106-117, 2004.

FRIED, L. P. et al. Building communities that promote successful aging. West. J. Med., v. 167, n. 4, p. 216-219, 1997.

GARDNER, P. J. Natural neighborhood networks: important social networks in the lives of older adults aging in place. J. Aging Stud., v. 25, n. 3, p. 263-271, 2011. 
GASPARI, V. P.; BOTEGA, N. J. Rede de apoio social e tentativa de suicídio. J Bras Psiquiatr., v. 51, p. 233-240, 2002.

GEIB, L. T.C. Determinantes sociais da saúde do idoso. Ciência \& Saúde Coletiva, v. 17, n. 1, p. 123-133, 2012.

GOMIDE, M.; SCHÜTZ, G. E. Análise de Redes Sociais e práticas avaliativas: desafios à vista. Physis Revista de Saúde Coletiva. Rio de Janeiro, v. 25, n. 3, p. 819-842, 2015.

GONÇALVES, T. R. et al. Avaliação de apoio social em estudos brasileiros: aspectos conceituais e instrumentos. Ciênc. Saúde Coletiva, v. 16, n. 3, p. 1755-1769, 2011.

GRIEP, R. H. et al. Validade de construto de escala de apoio social do Medical Outcomes Study adaptada para o português no Estudo Pró-Saúde. Cad Saude Publica, v. 21, p. 703714, 2005.

HALE, J. Connecting food environments and health through the relational nature of aesthetics: gaining insight through the community gardening experience. Soc. Sci. Med., v. 72, n. 11, p. 1853-1863, 2011.

HOBBS, W. R. et al. Online social integration is associated with reduced mortality risk. PNAS, p. 1-5, abr., 2016.

JAMES, B. D. et al. A. Relation of late-life social activity with incident disability among community-dwelling older adults. J. Gerontol., v. 66, n. 4, p. 467-473, 2011.

JOHNSON, E. R. et al. Relationship between social support and body mass index among overweight and obese African American women in the rural deep south, 2011-2013. Prev Chronic Dis, v. 11, p. 14-34, 2014.

KENDIG, H.; BROOKE, L. Australian research on ageing and social support. Aust. J. Ageing, v. 16, n. 3, p. 127-130, 1997.

KIM, S. H. Effects of a Volunteer-Run Peer Support Program on Health and Satisfaction with Social Support of Older Adults Living Alone. J Korean Acad Nurs, v. 42, n. 4, p. $525-$ 536, 2012.

KLEIMAN, E. M.; LIU, R. T. Social support as a protective factor in suicide: Findings from two nationally representative samples. J Affect Disord., v. 150, n. 2, p. 540-545, 2014.

KRISTJANSSON, B.; BREITHAUPT, K.; MCDOWELL, I. Development and validation of an indicator of support for community-residing older Canadians. Int. Psychogeriatr., supl. 1, v. 13, p. 125-135, 2001.

KUTEK, S. M.; TURNBULL, D.; FAIRWEATHER-SCHMIDT, A. K. Rural men's subjective well-being and the role of social support and sense of community: evidence for the potential benefit of enhancing informal networks. Aust. J. Rural Health, v. 19, n. 1, p. 20-26, 2011.

LADITKA, J. N. et al. Promoting cognitive health: a formative research collaboration of the healthy aging research network. Gerontologist, supl. 1, v. 49, p. S12-S17, 2009. 
LARSSON, K.; THORSLUND, M. Does gender matter? Differences in patterns of informal support and formal services in a swedish urban elderly population. Res. Aging., v. 24, n. 3, p. 308-336, 2002.

LETCHER, A. S.; PERLOW, K. M. Community-based participatory research shows how a community initiative creates networks to improve well-being. Am. J. Prev. Med., supl. 1, v. 37, n. 6, p. S292-299, 2009.

MAIA, C. M. L et al. Redes de apoio social e de suporte social e envelhecimento ativo. International Journal of Developmental and Educational Psychology INFAD. Revista de Psicología, v. 1, n. 1, p. 293-303, 2016.

MARQUES, C. A. et al. Associação entre depressão, níveis de dor e falta de apoio social em pacientes internados em enfermarias de clínica médica. J Bras Psiquiatr, v. 62, n. 1, p. 1-7, 2013.

MARTINS, R. M. L. A relevância do apoio social na velhice. Educação, ciência e tecnologia. 2005. Disponível em: <http://www.ipv.pt/Millenium/Millenium31/9.pdf>. Acesso em: out 2017.

MEDINA-WALPOLE, A. et al. The current state of geriatric medicine: a national survey of fellowship-trained geriatricians, 1990-1998. J. Am. Geriatr. Soc., v. 50, n. 5, p. 949-955, 2002.

MELCHIORRE, M. G. et al. Social support, socio-economic status, health and abuse among older people. Seven European Countries., v. 8, issue 1, e54856, January 2013.

MENDES, E. V. As redes de atenção à saúde. Ciência \& Saúde Coletiva, v. 15, n. 5, p. $2297-$ $2305,2010$.

MUI, A. C. Depression among elderly Chinese inmigrants: an exploratory study. Soc. Work., v. 41, n. 6, p. 633-645, 1996.

NERI, A. L.; VIEIRA, L. A. M. Envolvimento social e suporte social percebido na velhice. Rev. Bras. Geriatr. Gerontol. Rio de Janeiro, v. 16, n. 3, p. 419-432, 2013.

ORGANIZAÇÃO MUNDIAL DE SAÚDE. Preventing suicide: a global imperative. Geneva: WHO, 2014.

PAIM, J. S. et al. O sistema de saúde brasileiro: história, avanços e desafios. The Lancet, p. 11-31, maio 2011.

PAIM, J. S.; ALMEIDA FILHO, N. Reforma Sanitária Brasileira em perspectiva e o SUS. In: . Saúde Coletiva: teoria e prática. Rio de Janeiro: MedBook, 2014. p. 13-27.

PAZIN, J. et al. Atividade física no lazer, deslocamento, apoio social e percepção do ambiente urbano em homens e mulheres de Florianópolis/SC. Rev Bras Educ Fís Esporte. São Paulo, v. 30, n. 3, p. 743-55, jul.-set., 2016. 
PESSINI, J. et al. Avaliação da equivalência transcultural e de propriedades psicométricas da escala de apoio social para hábitos alimentares saudáveis. Rev. Nutr., Campinas, v. 29, n. 6, p. 797-807, nov./dez., 2016.

RAMOS, M.P. Apoio social e saúde entre idosos. Sociologias, v. 4, n. 7, p. 156-175, 2002.

REIS, M.S. et al. Validade e fidedignidade de uma escala de avaliação do apoio social para a atividade física. Rev Saúde Pública. São Paulo, v. 45, n. 2, p. 294-301, 2011.

RODRIGUES, A .G.; SILVA A. A. A rede social e os tipos de apoio recebidos por idosos institucionalizados. Rev. Bras. Geriatr. Gerontol. Rio de Janeiro, v. 16, n. 1, p. 159-170, 2013. SEHLO, M.G.; KAMFAR, H.Z. Depression and quality of life in children with sickle cell disease: the effect of social support. BMC Psychiatry, v. 15, p. 78, 2015.

SEIDL, E.M.; TRÓCCOLI, B .T. Desenvolvimento de escala para avaliação do suporte social em HIV/Aids. Psicol Teor Pesqui, v. 22, p. 317-326, 2006.

SIQUEIRA, M. M. M. Construção e validação da escala de percepção de suporte social. Psicologia em Estudo. Maringá, v. 13, n. 2, p. 381-388, abr./jun. 2008.

STAMM, T. Education, graduate and continuing in geriatrics and geriatric rehabilitation. Z. Gerontol. Geriatr., supl. 1, v. 34, p. 43-48, 2001.

THANAKWANG, K. et al. Mechanisms by which social support networks influence healthy aging among Thai community-dwelling elderly. J. Aging Health, v. 23, n. 8, p. 1.352-1.378, 2011.

WALTER-GINZBURG, A. Social factors and mortality in the old-old in Israel: the CALAS study. J. Gerontol., v. 57, n. 5, p. S308-S318, 2002.

WU, Z.; POLLARD, M. S. Social support among unmarried childless elderly persons. J. Gerontol., v. 53, n. 6, p. S324-S335, 1998.

ZUNZUNEGUI, M. V. et al. Social networks and self-rated health in two French-speaking Canadian community dwelling populations over 65. Soc. Sci. Med., v. 58, n. 10, p. 2.069$2.081,2004$.

\section{Nota}

${ }^{1}$ M.B.O.G. Guedes foi responsável pela fundamentação teórica, elaboração, revisão e formatação do texto. K.C. Lima, C.P. Caldas e R.P. Veras responsabilizaram-se pela fundamentação teórica, elaboração e revisão do artigo. 


\section{Abstract}

\section{Social support and comprehensive health care for the elderly}

The growing population aging and socio-cultural changes of our time lead to new needs, not only care, but also to meet a new perspective of the concept of health care in which the disease has become of limited action. This study is an essay based on the scientific literature and proposes to think about a small part of this great question: social support as part of comprehensive health care for older persons. For this, a narrative review of the literature was made in Pub Med / MedLine, Crochane and Scopus databases, as well as in the reference lists of the articles found. It is part of understanding the role of social support and micro social networks on the health of older persons. Next, we discuss ways to assess the support and the social network and reflect on the role of social support in the Unified Health System. Finally, it approaches the management of social support. As final considerations the main challenges for the social support is actually considered as a determinant of human health are highlighted.

> Keywords: social support; elderly people's health; integrality in health. 